\title{
Anatomy of the facial nerve branching patterns, the marginal mandibular branch and its extraparotid ramification in relation to the lateral palpebral line
}

\author{
Ekaritt Weerapant ${ }^{\mathrm{a}}$, Tanom Bunaprasert ${ }^{\mathrm{b}}$, Prayuth Chokrungvaranont ${ }^{\mathrm{c}}$, Vilai Chentanez ${ }^{\mathrm{d}}$ \\ ${ }^{a}$ Master of Science Program in Medical Science, ${ }^{b}$ Department of Otolaryngology, ${ }^{c}$ Department of \\ Surgery, ${ }^{d}$ Department of Anatomy, Faculty of Medicine, Chulalongkorn University, Bangkok 10330, \\ Thailand
}

\begin{abstract}
Background: Surgery of face and parotid gland may cause injury to branches of the facial nerve, which results in paralysis of muscles of facial expression. Knowledge of branching patterns of the facial nerve and reliable landmarks of the surrounding structures are essential to avoid this complication.

Objective: Determine the facial nerve branching patterns, the course of the marginal mandibular branch (MMB), and the extraparotid ramification in relation to the lateral palpebral line (LPL).

Materials and methods: One hundred cadaveric half-heads were dissected for determining the facial nerve branching patterns according to the presence of anastomosis between branches. The course of the MMB was followed until it entered the depressor anguli oris in 49 specimens. The vertical distance from the mandibular angle to this branch was measured. The horizontal distance from the LPL to the otobasion superious (LPL-OBS) and the apex of the parotid gland (LPL-AP) were measured in 52 specimens.

Results: The branching patterns of the facial nerve were categorized into six types. The least common (1\%) was type I (absent of anastomosis), while type V, the complex pattern was the most common (29\%). Symmetrical branching pattern occurred in $30 \%$ of cases. The MMB was coursing below the lower border of the mandible in $57 \%$ of cases. The mean vertical distance was $0.91 \pm 0.22 \mathrm{~cm}$. The mean horizontal distances of LPL-OBS and LPLAP were $7.24 \pm 0.6 \mathrm{~cm}$ and $3.95 \pm 0.96 \mathrm{~cm}$, respectively. The LPL-AP length was $54.5 \pm 11.4 \%$ of LPL-OBS.

Conclusion: More complex branching pattern of the facial nerve was found in this population and symmetrical branching pattern occurred less of ten. The MMB coursed below the lower border of the angle of mandible with a mean vertical distance of one centimeter. The extraparotid ramification of the facial nerve was located in the area between the apex of the parotid gland and the LPL.
\end{abstract}

Keywords: Branching patterns, facial nerve, lateral palpebral line, marginal mandibular branch, parotid gland

The facial nerve emerges from the skull through the stylomastoid foramen. It immediately gives off the posterior auricular nerve to supply the auricularis posterior and occipital belly of the occipitofrontalis muscles [1].Then, the main trunk of the facial nerve runs within the parenchyma of the parotid gland and divides into two main divisions, the temporofacial and

Correspondance to: Vilai Chentanez, MD, PhD, Department of Anatomy, Faculty of Medicine, Chulalongkorn University, Bangkok 10330, Thailand. E-mail: fmedvct@md.chula.ac.th; cvilai1@yahoo.com cervicofacial divisions, at the posterior border of the ramus of the mandible. Each division is further divided into several branches in a plexiform arrangement, giving off five terminal branches including the temporal, zygomatic, buccal, marginal mandibular and cervical branches, which distribute over the face and the upper neck to supply the muscle of facial expression. Davis [2] reviewed 350 cervicofacial halves and categorized six types of facial nerve patterns within parotid gland. The branching patterns of the facial nerve were also investigated by 
several other studies $[3,4]$. The percentage of each type in those studies was different as shown in Table 1. Therefore, the branching patterns of the facial nerve after exiting the stylomastoid foramen and ramifying on the face were determined and categorized based on the presence of anastomosis within each division and between divisions [2]. Subsequent studies used the inferior border and angle of the mandible to locate the marginal mandibular branch (MMB), which might be useful for a submandibular surgical approach [5-11]. Then, the anatomic relationship of the MMB to the inferior border of the mandible was investigated. Surgical landmarks of the facial nerve trunk for parotid gland surgery have been investigated in both cadaveric dissection and intraoperative measurements [12-14]. Until now, there is no conclusive evidence for the best single landmark. Here, the lateral palpebral line (LPL), a vertical line passing the lateral palpebral angle (the outer corner of the eye fissure where the eyelids meet), parallel to the mid sagittal plane of the face, was introduced as a reference line for surgical landmark of facial nerve ramification. The distances from the LPL to the otobasion superious (the highest point of attachment of the external ear to the head) (LPL-OBS) and to the apex of the parotid gland (the point where the anterior border met the superior border of the gland) (LPL-AP) were determined in order to locate the area of facial nerve branching in relation to the LPL.

\section{Materials and methods}

One hundred halves of embalmed adult cadaver faces from the Department of Anatomy, Faculty of Medicine, Chulalongkorn University, Bangkok, Thailand, were dissected. The reference points were marked by putting the pins into the lateral palpebral angle and the OBS. The skin of the face was carefully removed, the boundary of the parotid gland was identified, and the apex of the parotid gland (AP) was marked by a pin. Here, the AP was used as a landmark for the extraparotid ramification of the facial nerve.
After the branches of facial nerve were identified medially (anteriorly) to the apex of the parotid gland, this gland was carefully removed and then the nerve was followed proximally to the stylomastoid foramen. The two primary divisions of the facial nerve were exposed. Small anastomotic branches between the terminal branches of the facial nerve were carefully dissected.

\section{Branching patterns of the facial nerve}

Photographs of each specimen were taken and the branching pattern was drawn as shown in Fig. 1.

The branching patterns of 100 specimens were classified into six types based on the description of Davis [2] as follows:

(Type I) no anastomosis occurred between branches of the facial nerve.

(Type II) presence of an anastomotic connection between branches of temporofacial division.

(Type III) a single anastomosis between the temporofacial and cervicofacial divisions

(Type IV) a combination of type II and type III.

(Type V) two anastomotic rami passed from the cervicofacial division to interwine with the branches of temporofacial division.

(Type VI) plexiform arrangement, the mandibular branch sent twig to join any members of the temporofacial division.

The percentage of each type was calculated.

\section{The marginal mandibular branch}

The MMB was dissected along the inferior border of the mandible until it crossed the facial artery and entered the depressor anguli oris muscle in 49 specimens. The course of this nerve and its relation to the inferior border of the mandible was recorded. If this branch dropped below the inferior border of the mandibular angle, then the vertical distance from the lower end of mandibular angle was measured as shown in Fig. 2. The mean, standard deviation (SD) and range of each measurement were assessed.

Table 1. Percentage of facial nerve branching patterns in each type from previous and the present studies.

\begin{tabular}{lccccccc}
\hline \multirow{2}{*}{ Author (s) } & $\begin{array}{l}\text { Number } \\
\text { of case (s) }\end{array}$ & \multicolumn{5}{c}{ Type (\%) } \\
\cline { 3 - 7 } & & I & II & III & IV & V & VI \\
\hline Davis et al. [2] & 350 & 13 & 20 & 28 & 24 & 9 & 6 \\
Bernstein et al. [3] & 35 & 9 & 9 & 25 & 19 & 22 & 16 \\
Myint et al. [4] & 79 & 11 & 16 & 34 & 19 & 7 & 13 \\
The present study & 100 & 1 & 10 & 20 & 18 & 29 & 21 \\
\hline
\end{tabular}


A

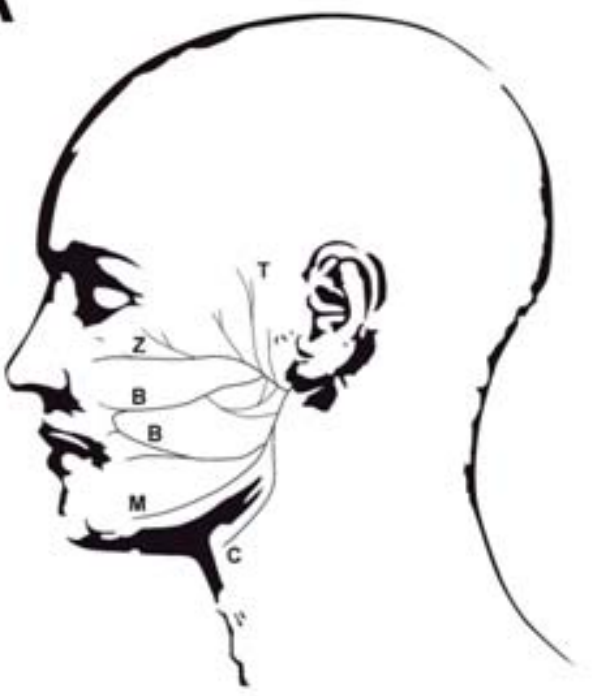

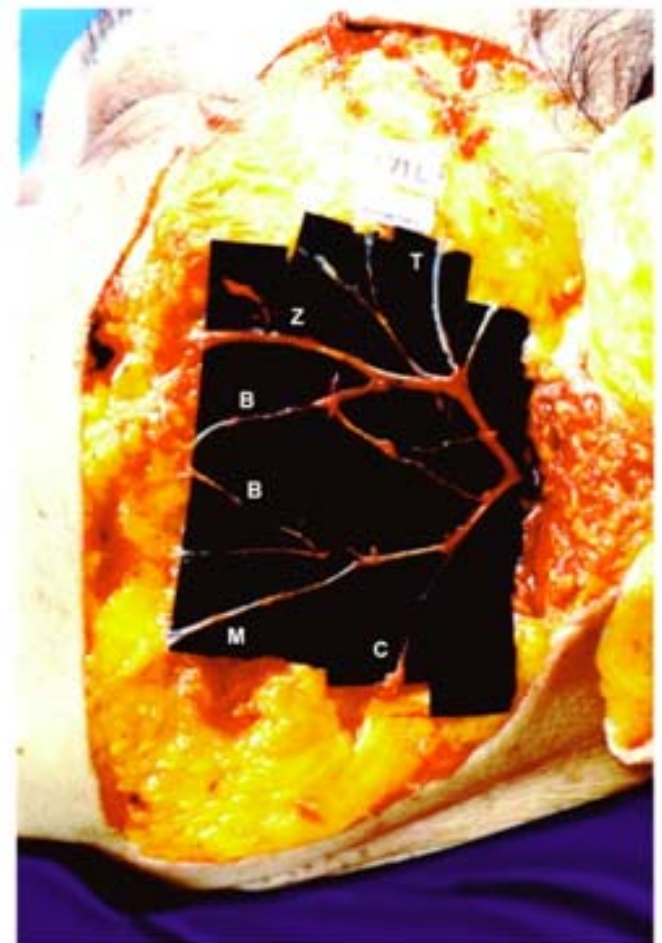

B

Fig. 1 Facial nerve branching (A) schematic drawing (B) on cadaver. $\mathrm{T}=$ temporal branch, $\mathrm{Z}=$ zygomatic branch, $\mathrm{B}=$ buccal branch, $\mathrm{M}=$ marginal mandibular branch, $\mathrm{C}=$ cervical branch .

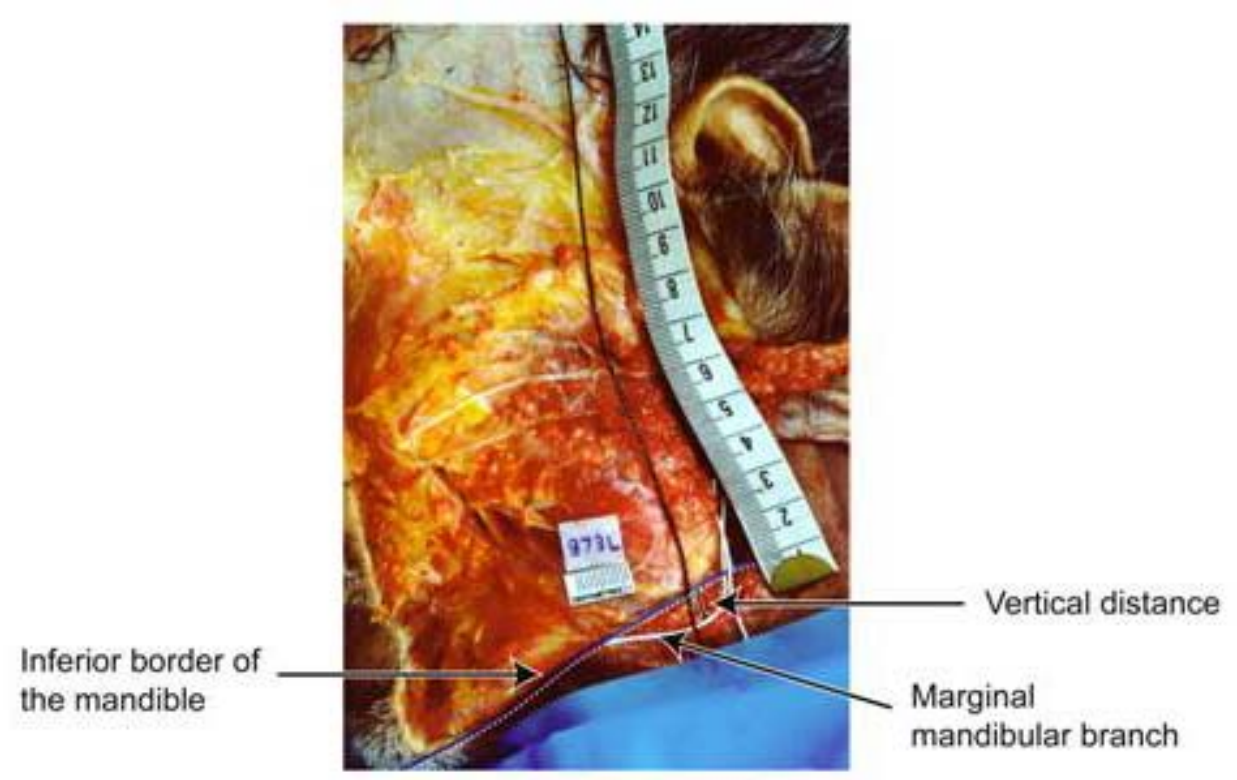

Fig. 2 Photograph of the half cadaveric face showing the relation between marginal mandibular branch and inferior border of the mandible. 


\section{Measurement of the distances from the lateral palpebral line}

Fifty-two specimens with complete dissection were measured. A vertical line passing the lateral palpebral angle and parallel to the mid sagittal plane of the face was marked by a thread, which represented the LPL as shown in Fig. 3. The second and third vertical line passing the OBS and the AP were also marked (line A and line B in Fig. 3, respectively). The horizontal distance between LPL and the OBS (LPL-OBS) was measured by a flexible measuring tape. Then, the horizontal distance between the LPL and AP (LPL-AP) was also measured. All measurements were performed by one examiner. Each measurement was done in three times and the mean was calculated. The mean and standard deviation of all measurements including the proportion of LPL-OBS to LPL-AP were calculated.

\section{Results}

\section{Branching patterns}

The branching patterns of the facial nerve from 100 specimens were classified into six types according to the presence of anastomosis between its branches and divisions. More complex patterns were found in this study. The percentage of each type was type I (1\%), type II (10\%), type III (20\%), type IV (18\%), type V (29\%) and type VI (21\%). Symmetrical branching pattern was found in 15 cadavers (30\% of cases).There were one case in type II and type IV, three cases in type III and type VI, and seven cases in type V. The least common was type I. The present result is compared with those in previous studies in Table 2.

\section{The marginal mandibular branch}

The course of the MMB was followed until it entered the depressor anguri oris in 49 specimens. Posterior to the facial artery, the nerve passed above the inferior border of the mandible in 28 cases (57\%). It was observed that, when the nerve passed anteriorly to the facial artery, it turned up to be above the inferior mandibular border. The vertical distance from the MMB to the inferior margin of the mandible at the mandibular angle (Fig.2) ranged from 0.50-1.35 cm with a mean of $0.91 \pm 0.22 \mathrm{~cm}$. (Table 2).

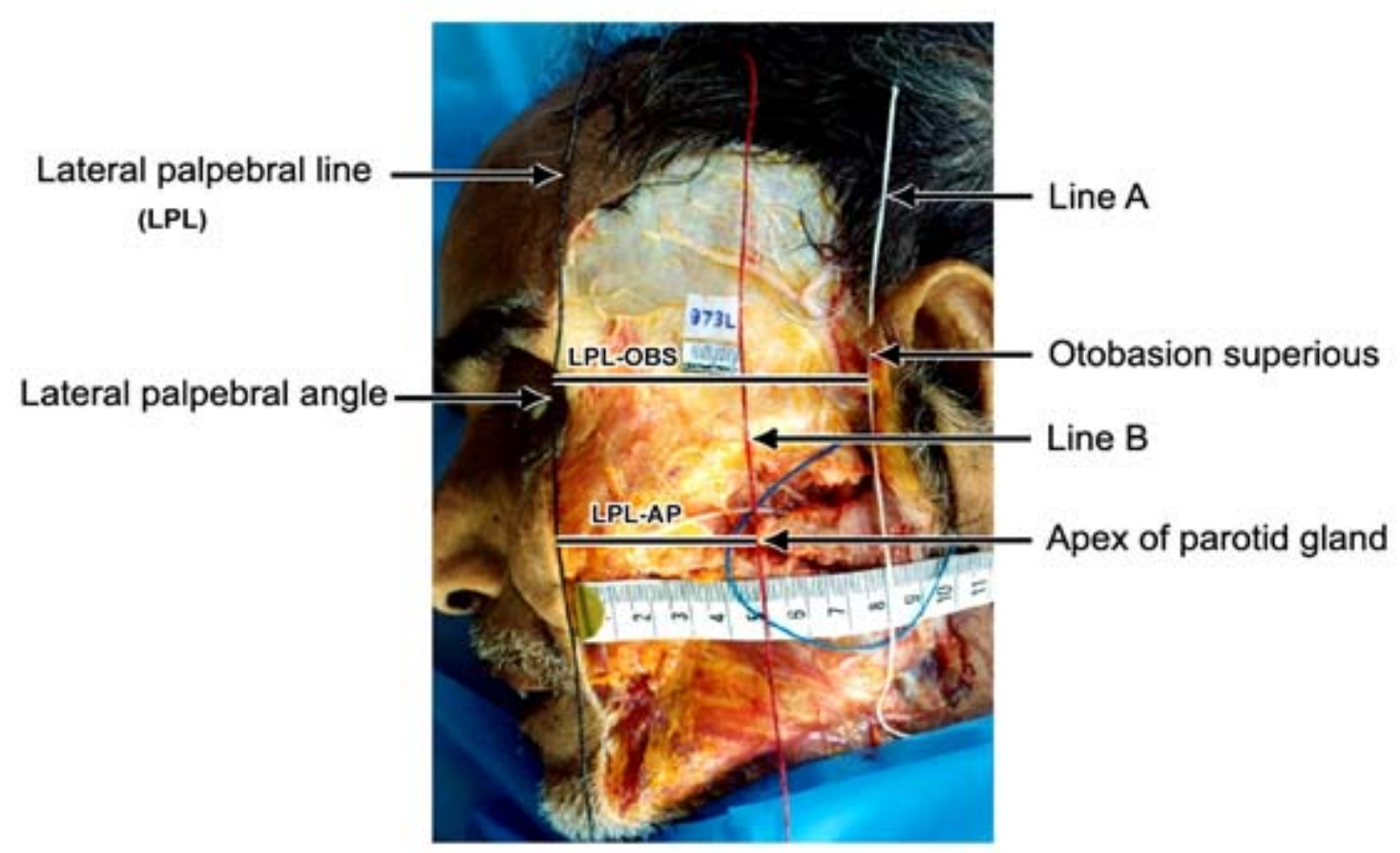

Fig. 3 Photograph of the half-cadaveric face showing the surgical landmarks including the lateral palpebral angle, the lateral palpebral line (LPL), the otobasion superious (OBS) and the apex of the parotid gland (AP). Line A and Line B represent the vertical line passing the OBS and apex of the parotid gland, respectively. LPL-OBS= distance from the LPL to OBS. LPL-AP= distance from the LPL to AP. 
Table 2. The marginal mandibular branch and its relation to the inferior border of the mandible.

\begin{tabular}{|c|c|c|c|c|}
\hline \multirow[t]{3}{*}{ Author (s) } & \multirow{3}{*}{$\begin{array}{l}\text { Number } \\
\text { of case(s) }\end{array}$} & \multicolumn{3}{|c|}{ Relation to the inferior margin of mandible } \\
\hline & & Above & Below & \\
\hline & & Number (\%) & Number (\%) & Distance (cm) \\
\hline Dingman and Grabb [5] & 100 & 81 & 19 & 1.0 \\
\hline Ziarah and Atkinson [6] & 110 & 47 & 53 & 1.2 \\
\hline Wang et al. [7] & 120 & 67 & 33 & 2.5 \\
\hline Woltmann et al. [10] & 45 & 58 & 43 & 1.2 \\
\hline Saylam et al. [11] & 50 & 74 & 26 & - \\
\hline The present study & 49 & 57 & 43 & 0.9 \\
\hline
\end{tabular}

\section{Distances from the LPL}

The LPL-OBS and LPL-AP distances were measured in 52 specimens. The range and mean of these measurements were shown in Table 3 . The LPL-OBS and LPL-AP distances were 7.27 \pm 0.60 and $3.95 \pm 0.96 \mathrm{~cm}$, respectively. The proportion of LPLAP to LPL-OBS was $54.5 \pm 11.4 \%$. This was approximately one-half of the LPL-OBS distance.

\section{Discussion}

Various surgical landmarks of the facial nerve have been investigated to allow for a safer and quicker parotidectomy or other surgery in the lateral face. Surface landmarks for the facial nerve trunk have been proposed. Pather and Osman [14] suggested the use of the transverse process of the axis as the landmark for the facial nerve trunk because it was easily palpated, did not require a complex dissection, and had low risk of injury to the facial nerve trunk. In addition, intraoperative measurement of the facial nerve trunk from the reference point nerve $(10 \mathrm{~mm}$ proximal to its bifurcation) to the skin surface and the summit of the tragus were obtained [13]. This information was suggested to be helpful in the mobilization of the parotid gland from its posterior relations. Tragus and the ala nasi were also used as superficial anatomic landmarks for buccal branches of the facial nerve [11]. In all specimens $(n=60)$, the buccal branches were coursing below the line that joined the tragus and ala nasi. These branches of the facial nerve were located in the lateral face. It is vulnerable to surgical injury especially in the mobilization of the parotid gland. The present study showed a more complex branching pattern of the facial nerve when compared to previous studies shown in Table 1. Type V was the most common (29\%). Only one case of type I was found in this study. Type I was important clinically, since if any branch was sacrificed, persistent paralysis of the muscle would develop. In addition, recovery of the facial muscle paralysis could occur in the more complex branching pattern. A symmetrical branching pattern was found in 15 cadavers (30\% of cases). Occurrences of the same type of facial nerve branching pattern bilaterally in previous studies $[15,16]$ were more frequent $(47 \%$ and 53\%). Hence, the surgeon must be aware that the same branching pattern can not be assumed on both sides.

The relation of the MMB to the inferior border of the mandible in the present study was consistent with that of Woltmann [10], but was different from other studies [5-7, 11] (Table 2). In 43\% of cases, the nerve was found inferior to the inferior margin of the mandible. The mean distance recorded in the present study was $1 \mathrm{~cm}$, which was in agreement with most of the previous investigations except for Wang et al. [7], $(2.1-3.0 \mathrm{~cm})$. The present data confirmed the previous suggestion to avoid this nerve injury when performing surgery of the mandible by making an incision at least $2 \mathrm{~cm}$ below the inferior margin of the

Table 3. Horizontal Distance from the lateral palpebral line to the otobasion superious (LPL-OBS) and apex of the parotid gland (LPL-AP).

\begin{tabular}{lccc}
\hline Measurement & Number of cases & Range $(\mathbf{c m})$ & Mean \pm SD $(\mathbf{c m})$ \\
\hline LPL-OBS & 52 & $6.15-8.8$ & $7.24 \pm 0.60$ \\
LPL-AP & 52 & $1.65-6.5$ & $3.95 \pm 0.96$ \\
\hline
\end{tabular}


mandible $[5,8,10]$. However, the dissections in the present study were performed on embalmed cadavers. The tissues were stiff, contracted and shrunken when compared to the fresh specimen. Therefore, difference between the results of the present study concerning the distances and those of the previous studies might be due to the fixation. In elderly with loose skin, the MMB might be below the lower border of the mandible more than $2 \mathrm{~cm}$.

The present study used the LPL as a reference identification of the ramification of the facial nerve on the face. It is practical and easy to locate this line. In order to study the extraparotid ramification of the facial nerve, the AP was also used as another landmark. The result of measurement demonstrated that the LPL-AP was 55\% of LPL-OBS. Therefore, the AP seemed to be located at the midpoint of LPLOBS. It might be indicated that the location of the branches of facial nerve after emerging from the parotid gland was approximately half of the distance between the LPL and the OBS (Fig. 3). These anatomical data are necessary for a surgeon to know to be able to carefully dissect and preserve the facial nerve during lateral face surgery.

In conclusion, this cadaveric study investigated the branching pattern of facial nerve in Asian race. More complex branching pattern but less symmetry was demonstrated. The MMB passed under the inferior margin of the mandible in $43 \%$ of cases and the mean lowest point was $0.91 \pm 0.22 \mathrm{~cm}$. The facial nerve gave off branches after emerging from the parotid gland at the midpoint of LPL-OBS. These findings are essential for avoiding injury to the branches of facial nerve during surgery on the lateral face.

\section{Acknowledgements}

The authors would like to thank Mr. Veerachai Kongwan for his assistance in taking the photographs.

The authors have no conflict of interest to report.

\section{List of abbreviation}

AP = apex of parotid gland,

LPL = lateral Palpebral Line,

$\mathrm{MMB}=$ marginal mandibular branch,

OBS $=$ otobasion superious.

\section{References}

1. Moore KL, Dalley AF. Clinical Oriented Anatomy. 9th ed. Baltimore:Lippincott Williams and Wilkins; 1999. p. 862-4.
2. Davis RA, Anson BJ, Budinger JM, Kurth LR. Surgical anatomy of the facial nerve and parotid gland based upon a study of 350 cervicofacial halves. Surg Gynecol Obstet. 1956; 102:385-413.

3. Bernstein L, Nelson RH. Surgical anatomy of the extraparotid distribution of the facial nerve. Arch Otolaryngol. 1984; 110:177-83.

4. Myint K, Azian AL, Khairul FA. The clinical significance of the branching pattern of the facial nerve in Malaysian subjects. Med J Malaysia. 1992; 47: 114-21.

5. Dingman RO, Grabb WC. Surgical anatomy of the mandibular ramus of the facial nerve based on the dissection of 100 facial halves. Plast Reconstr Surg. 1962; 29:266-72.

6. Ziarah HA, Atkinson ME. The surgical anatomy of the mandibulardistribution of the facial nerve. Br J Oral Surg. 1981;19:159-70.

7. Wang TM, Lin CL, Kuo KJ, Shih C. Surgical anatomy of the mandibular ramus of the facial nerve in Chinese adults. Acta Anat (Basel). 1991; 142:126-31.

8. Basar R, Surgon MF, Tekdemir I, Elhan A. The marginal mandibular branch of the facial nerve. Surg Radiol Anat. 1997; 19:311-4.

9. Potgieter W, Meiring JH, Boon JM, Pretorius E, Pretorius JP, Becker PJ. Mandibular landmarks as an aid in minimizing injury to the marginal mandibular branch: a metric and geometric anatomical study. Clin Anat. 2005; 18:171-8.

10. Woltmann M, Faveri R, Sgrott EA. Anatomosurgical study of the marginal mandibular branch of the facial nerve for submandibular surgical approach. Braz Dent J. 2006; 17:71-4.

11. Saylam C, Ulcerler H, Orhan M. Anatomic landmarks of the buccal branches of the facial nerve. Surg Radiol Anat. 2006; 28:462-7.

12. de Ru JA, Bleys RL, van Benthem PP, Hordijk GJ. Preoperative determination of the location of parotid gland tumors by analysis of the position of the facial nerve. J Oral Maxillofac Surg. 2001; 59:525-8.

13. Wong DS. Surface landmarks of the facial nerve trunk: a prospective measurement study. ANZ J Surg. 2001; 71:753-6.

14. Pather N, Osman M. Landmarks of the facial nerve: implications for parotidectomy. Surg Radiol Anat. 2006; 28:170-5.

15. Kopuz C, Turgut S, Yavuz S, Ilgi S. Distribution of facial nerve in parotid gland: analysis of 50 cases. Okajimas Folia Anat Jpn. 1994; 70:295-9.

16. Ekinci N. A study on the branching pattern of the facial nerve of children (in Japanese). Kaibogaku Zasshi. 1999; 74:447-50. 